\title{
Optimization Using a New Bio-inspired Approach
}

\author{
Xiang Feng ${ }^{1}$, Francis C.M. Lau $^{2}$, and Daqi Gao ${ }^{1}$ \\ ${ }^{1}$ East China University of Science and Technology, Shanghai 200237, China \\ $\{$ xfeng, gaodaqi $\}$ @ecust.edu.cn \\ 2 The University of Hong Kong, Hong Kong \\ f cmlau@cs.hku.hk
}

\begin{abstract}
There is growing interest in bio(logy)-inspired approaches that are inspired by the principles of biology and that can solve difficult problems. In this paper, we propose a new computational algorithm that is inspired by molecular mechanics for the solution of complex problems. There is a deep and useful connection between mechanics mechanics and combinatorial optimization. This connection exposes new information and allows an unfamiliar perspective on traditional optimization problems and approaches. The alternative of molecular mechanics algorithm (MMA) to traditional approaches has the advantages of inherent parallelism and the ability to deal with a variety of complicated social interactions, autonomous behaviors and multiple objectives.
\end{abstract}

Keywords: Bio-inspired algorithm, multi-objective optimization, molecular mechanics algorithm (MMA), molecular dynamics.

\section{Introduction}

Many artifacts have been built throughout history, and many of which obtained their inspiration from phenomena in the natural world. It is noted that "progress often occurs at the boundaries between disciplines." [1] In the field of computer science, especially in artificial intelligence, there is growing interest in paralleldistributed intelligent theories and approaches that are inspired by the principles of nature and that can solve difficult problems. Bio(logy)-inspired approaches are probably the best known example of such nature-inspired approaches. Successful bio-inspired approaches include Genetic Algorithm (1975) [2, Ant Colony Optimization (1991) [3,4, and Particle Swarm Optimization (1995) 5]. There were also physics-inspired approaches such as Simulated Annealing Algorithm (1983) 6. The fields of biology and physics have flourished in a rich soil for many years. We believe there exist many opportunities for the application of the principles in biology and physics to computing. This paper proposes a brand new bio-inspired approach for solving difficult computational problems.

J. Zhou (Ed.): Complex 2009, Part I, LNICST 4, pp. 39 2009.

(C) ICST Institute for Computer Sciences, Social Informatics and Telecommunications Engineering 2009 


\section{Molecular Mechanics Algorithm (MMA)}

The distribution problem is one of those difficult computational problems. The mathematical structure of the distribution problem is simple. The distribution problem defined below is a typical NP-hard combinatorial optimization problem.

Definition 1. In a multi-objective framework, the distribution problem can be formulated as

$$
\left\{\begin{array}{l}
\max : \\
z^{q}(R)=\left(C^{q}\right)^{T} X(R)^{T}=\sum_{i=1}^{I} \sum_{j=1}^{J} c_{i j}^{q} x_{i j} r_{i j} \quad q=1, \cdots, Q \\
\text { s.t. } \quad \sum_{j=1}^{J} r_{i j}=1 \quad i=1, \cdots, I \\
x_{i j}=0,1
\end{array}\right.
$$

where $q$ represents the objective, $R$ is a two-dimensional distribution vector, $C^{q}$ is a two-dimensional weight vector $(q=1,2, \cdots, Q)$, and $X$ is a two-dimensional Boolean vector.

With this problem model, we can now examine the bio-inspired multiobjective model which can mathematically describe an MMA. The theory of evolution behind the model is a dynamical theory.

The bio-inspired dynamics will drive the MMA to its equilibrium state.

Definition 2. The distribution and weight dynamic equations of the MMA are defined, respectively, by

$$
\begin{aligned}
& r_{i j}(t+1)=r_{i j}(t)+\Delta r_{i j}(t) \\
& c_{i j}^{q}(t+1)=c_{i j}^{q}(t)+\Delta c_{i j}^{q}(t)
\end{aligned}
$$

The two dynamic equations are seen as the "MMA evolution" by fictitious agents (molecules) which manipulate the distribution and weight vectors until an equilibrium is reached. In the MMA, every entry of distribution vector $R$ is treated as a fictitious agent (molecule). In fact, the weight vector is invariable, and the evolution of the weight vector only occurs in the computing process in order to obtain efficient solutions for the distribution vector.

For the fictitious agents - the molecules - there are four factors related to the evolutionary distribution vector $(\mathrm{R})$ and the weight vector $(\mathrm{C})$ :

- personal utility (u) (to realize the multiple objectives);

- whole utility $(\mathrm{J})$ (to increase the overall utility);

- minimal personal utility $(\mathrm{P})$ (to realize max-min fair distribution);

- interaction among the molecules (Q) (to satisfy the restrictions and to describe high-dimensional, highly nonlinear, random behaviors and dynamics). 
We try to solve the distribution problem defined in Definition 1 by subdividing this hard problem with respect to the four factors. In molecular mechanics, "energy" makes molecules move. When "energy" is equal to zero, the molecules will stop moving, being in an equilibrium state. For the MMA, we will define the "energy" function, which makes fictitious agents (molecules) evolve towards the optimum or until an equilibrium is reached. The "energy" function is based on the four factors each of which corresponds to a component of the "energy" function.

Definition 3. The "energy" function is defined by

$$
E_{i j}^{q}(t)=\lambda_{1} u_{i j}^{q}(t)+\lambda_{2} J^{q}(t)-\lambda_{3} P^{q}(t)-\lambda_{4} Q^{q}(t)
$$

where $0<\lambda_{1}, \lambda_{2}, \lambda_{3}, \lambda_{4}<1$. The larger the "energy" function, the faster the fictitious agent (molecule) would evolve towards the optimum.

According to "differential equation theory", a variable's increment to make it minimum is equal to the sum of negative items from related factors differentiating the variable. Because our defined problem is a "maximum" problem, a variable's increment to make it maximum is equal to the sum of the items from related factors differentiating the variable. Thus we have the following definitions.

Definition 4. The increments of distribution and weight are defined, respectively, by

$$
\begin{aligned}
& \Delta r_{i j}(t+1) \approx \frac{d r_{i j}(t)}{d t}=\frac{\partial E_{i j}^{q}(t)}{\partial r_{i j}(t)}=\sum_{q=1}^{Q}\left(\lambda_{1} \frac{\partial u_{i j}^{q}(t)}{\partial r_{i j}(t)}+\lambda_{2} \frac{\partial J^{q}(t)}{\partial r_{i j}(t)}-\lambda_{3} \frac{\partial P^{q}(t)}{\partial r_{i j}(t)}-\lambda_{4} \frac{\partial Q^{q}(t)}{\partial r_{i j}(t)}\right) \\
& \Delta c_{i j}^{q}(t+1) \approx \frac{d c_{i j}^{q}(t)}{d t}=\frac{\partial E_{i j}^{q}(t)}{\partial c_{i j}^{q}(t)}=\lambda_{1} \frac{\partial u_{i j}^{q}(t)}{\partial c_{i j}^{q}(t)}+\lambda_{2} \frac{\partial J^{q}(t)}{\partial c_{i j}^{q}(t)}-\lambda_{3} \frac{\partial P^{q}(t)}{\partial c_{i j}^{q}(t)}-\lambda_{4} \frac{\partial Q^{q}(t)}{\partial c_{i j}^{q}(t)} \\
& q=1,2, \cdots, Q
\end{aligned}
$$

Four kinds of factor functions in the "energy" function will be defined here, respectively.

Definition 5. The individual personal utility function for every agent (molecule) is defined by

$$
u_{i j}^{q}(t)=1-\exp \left(-c_{i j}^{q}(t) r_{i j}(t) x_{i j}(t)\right) \quad q=1,2, \cdots, Q
$$

Definition 6. The whole utility function for every agent (molecule) is defined by

$$
J^{q}(t)=\sum_{i=1}^{I} \sum_{j=1}^{J} u_{i j}^{q}(t) \quad q=1,2, \cdots, Q
$$


Definition 7. The gravitational potential energy function that makes minimal personal utility increase for every agent (molecule) is defined by

$$
\begin{aligned}
P^{q}(t) & =k^{2} \ln \sum_{i=1}^{I} \sum_{j=1}^{J} \exp \left[-\left(u_{i j}^{q}\right)^{2}(t) / 2 k^{2}\right]-k^{2} \ln I J \\
q & =1,2, \cdots, Q
\end{aligned}
$$

Definition 8. The interaction energy function is defined by

$$
Q^{q}(t)=\sum_{i=1}^{I}\left|\sum_{j=1}^{J} r_{i j}(t) x_{i j}(t)-1\right|^{2}-\sum_{i, j} \int_{0}^{u_{i j}^{q}}\left\{\left[1+\exp \left(-\zeta_{i j} x\right)\right]^{-1}-0.5\right\} d x
$$

where $\zeta_{i j}$ represents the intention strength of social coordination.

Now, we explain why the four kinds of functions are chosen.

1. Personal utility function. For the $q$-th objective, the larger the value of $c_{i j}^{q}(t) r_{i j}(t) x_{i j}(t)$ in Eq. (7), the more profit the $(i, j)$-th molecule gets. The optimization problem here is posed as a maximization problem. And we use the exponential function in order that $u_{i j}^{q}(t)$ would be between 0 and $1 . u_{i j}^{q}(t)$ can be regarded as the $q$-th dimensional utility of molecule. The larger $u_{i j}^{q}(t)$ is, the more profit the $(i, j)$-th molecule gets. Schematically, the $q$-th dimensional utility function $u_{i j}^{q}$ of a molecule corresponds to the $q$-th dimensional coordinate of the $(i, j)$-th molecule's $q$-th dimensional force field. We define the distance from the bottom boundary to the upper boundary of all of the molecule's $q$ dimensional force fields to be 1 . The biological meaning of the MMA will be discussed in Section IV. $1-e^{-x}$ is chosen as the definition of $u_{i j}^{q}$ because $1-e^{-x}$ is a monotonically increasing function and is between 0 and 1. Obviously, the larger $u_{i j}^{q}(t)$ the better.

2. Whole utility function. For this definition, we assume that the individual personal utilities are additive. Obviously, the larger $J^{q}(t)$ the better.

3. The potential energy function. For Eq. (9), $0<k<1$ is a parameter to be tuned in the implementation. The smaller $P^{q}$ is, the better. With Eq. (9), we attempt to construct a potential energy function, $P^{q}$, such that the decrease of its value would imply the increase of the minimal utility of all the molecules. We prove that in Theorem 1 . This way we can optimize the distribution problem in the sense that we consider not only the individual personal utility, but also the aggregate utilities, by increasing the minimum utility of all the molecules again and again. In fact, $k$ represents the strength of the upward gravitational force in the $q$-th dimensional force field. The bigger $k$ is, the faster the molecules would move up; hence, $k$ influences the convergence speed of the distribution problem. $k$ needs to be carefully adjusted in order to maximize the $q$ objectives.

4. The interaction energy function. For Eq. (10), the first term of $Q^{q}(t)$ is related to the constraints on capability; the second term involves social coordinations, with $\zeta_{i j}$ coming from Eqs. (11) - (13). If social coordinations are not involved in the system, $\zeta_{i j}$ will be a constant (e.g., 6 or 8 ). The first 
term of $Q^{q}(t)$ corresponds to a penalty function with respect to the constraint on the utilization of resources. The second term of $Q^{q}(t)$ is chosen as shown because we want $\frac{\partial Q^{q}}{\partial u_{i j}^{q}}$ to be a monotonically decreasing sigmoid function. $-\left\{\left[1+\exp \left(-\zeta_{i j} u_{i j}^{q}\right)\right]^{-1}-0.5\right\}$ is such a function. Therefore we let $\frac{\partial Q^{q}}{\partial u_{i j}^{q}}$ equal to $-\left\{\left[1+\exp \left(-\zeta_{i j} u_{i j}^{q}\right)\right]^{-1}-0.5\right\}$. Then $\frac{\partial Q^{q}}{\partial u_{i j}^{q}}$ is integrated to be $Q^{q}$.

$\zeta_{i j}(t) \uparrow \Rightarrow \frac{\partial Q^{q}}{\partial u_{i j}^{q}} \downarrow \Rightarrow-\frac{\partial Q^{q}}{\partial u_{i j}^{q}} \uparrow \stackrel{(5)}{\Rightarrow} \Delta r_{i j}(t+1) \uparrow \stackrel{a}{\Rightarrow} r_{i j}(t+1) \uparrow \stackrel{(7)}{\Rightarrow} u_{i j}^{q} \uparrow$

a: $r_{i j}(t+1)=r_{i j}(t)+\Delta r_{i j}(t+1)$

$\mathrm{b}: u_{i j}^{q}$ is a monotonically increasing function.

$$
\begin{gathered}
\zeta_{i j}(t)=\sum_{l=1}^{I} \zeta_{i l j}(t)+\sum_{l=1}^{I} \zeta_{l i j}(t) \\
\zeta_{i l j}(t)=\left\{\begin{array}{lll}
1 & \text { if } & \beta_{i l j} \in(I I) \cup(I V) \\
-1 & \text { if } & \beta_{i l j} \in(I) \cup(I I I)
\end{array}\right. \\
\zeta_{l i j}(t)=\left\{\begin{array}{lll}
1 & \text { if } & \beta_{l i j} \in(I) \cup(I V) \\
-1 & \text { if } & \beta_{l i j} \in(I I) \cup(I I I)
\end{array}\right.
\end{gathered}
$$

$\beta_{i l j}$ is the social coordination of agent $i$ with respect to agent $l$ for object $j$, which gives rise to the change $\zeta_{i l j}(t)$ of intention strength $\zeta_{i j}(t)$. The social coordination $\left(\beta_{i l j}\right)$ can be divided into four main categories as follows.

- I: Unilateral adaptive coordination

- II: Unilateral inducing coordination

- III:Bilateral adaptive coordination

- IV: Bilateral inducing coordination

We can therefore obtain the iteration speed by the following equation.

$$
\begin{aligned}
v_{i j}^{q} & =d u_{i j}^{q} / d t=\frac{\partial u_{i j}^{q}}{\partial r_{i j}} \frac{d r_{i j}}{d t}+\frac{\partial u_{i j}^{q}}{\partial c_{i j}^{q}} \frac{d c_{i j}^{q}}{d t} \\
& =\left[\lambda_{1}+\lambda_{2} \frac{\partial J^{q}(t)}{\partial u_{i j}^{q}(t)}-\lambda_{3} \frac{\partial P^{q}(t)}{\partial u_{i j}^{q}(t)}-\lambda_{4} \frac{\partial Q^{q}(t)}{\partial u_{i j}^{q}(t)}\right]\left\{\left[\frac{\partial u_{i j}^{q}(t)}{\partial r_{i j}(t)}\right]^{2}+\left[\frac{\partial u_{i j}^{q}(t)}{\partial c_{i j}(t)}\right]^{2}\right\}
\end{aligned}
$$

$v_{i j}^{q}$ represents the iteration speed of molecule $(i, j)$ (the $(i, j)$-th entry of distribution vector) with respect to the $q$-th objective. Meanwhile, $v_{i j}^{q}$ represents the speed of the upward movement of molecule $(i, j)$ in the $q$-th dimensional force field.

After having proposed the mathematical model of MMA, we give the parallel MMA in Table 1.

Proving the mathematical model of MMA

We now discuss the properties of the mathematical model of MMA as proposed above.

Theorem 1. If $k$ is very small, decreasing the potential energy function $P^{q}(t)$ of Eq. (9) amounts to increasing the minimal utility of molecules (entries in distribution vector $R$ ), minimized over $R$. 
Table 1. Algorithm MMA

\section{Input: $c_{i j}^{q}, x_{i j}, \zeta_{i j}$}

\section{Output:}

1. Initialization:

$$
\begin{aligned}
& t \leftarrow 0 \\
& r_{i j}(t)
\end{aligned}
$$

-Initialize in parallel

2. While $\left(d u_{i j}^{q} / d t \neq 0\right) \quad$ do

$$
t \leftarrow t+1
$$

$u_{i j}^{q}(t)$

$d u_{i j}^{q} / d t$

$d r_{i j}(t) / d t$

$r_{i j}(t) \leftarrow r_{i j}(t-1)+d r_{i j}(t) / d t$

$d c_{i j}^{q}(t) / d t$

$c_{i j}^{q}(t) \leftarrow c_{i j}(t-1)+d c_{i j}(t) / d t$
Compute in parallel according to Eq.(7)

- Compute in parallel according to Eq.(14)

-Compute in parallel according to Eq.(5)

-Compute in parallel according to Eq.(6)

Proof. Supposing that $H(t)=\max _{i, j}\left\{-\left(u_{i j}^{q}(t)\right)^{2}\right\}$, we have

$$
\left[\exp \left(H(t) / 2 k^{2}\right)\right]^{2 k^{2}} \leq\left\{\sum_{i=1}^{I} \sum_{j=1}^{J} \exp \left[-\left(u_{i j}^{q}(t)\right)^{2} / 2 k^{2}\right]\right\}^{2 k^{2}} \leq\left[I J \exp \left(H(t) / 2 k^{2}\right)\right]^{2 k^{2}}
$$

Taking the logarithm of both sides of the above inequalities gives

$$
H(t) \leq 2 k^{2} \ln \sum_{i=1}^{I} \sum_{j=1}^{J} \exp \left[-\left(u_{i j}^{q}(t)\right)^{2} / 2 k^{2}\right] \leq H(t)+2 k^{2} \ln I J .
$$

Since $I J$ is constant and $k$ is very small, we have

$$
H(t) \approx 2 k^{2} \ln \sum_{i=1}^{I} \sum_{j=1}^{J} \exp \left[-\left(u_{i j}^{q}(t)\right)^{2} / 2 k^{2}\right]-2 k^{2} \ln I J=2 P^{q}(t) .
$$

It turns out that the potential energy $P^{q}(t)$ at the time $t$ represents the maximum of $-\left(u_{i j}^{q}(t)\right)^{2}$ among all the molecules, which is the minimal personal utility of entries with respect to an objective $q$ at time $t$. Hence the decrease of potential energy $P^{q}(t)$ will result in the increase of the minimum of $u_{i j}^{q}(t)$.

Theorem 2. Updating the allotted entries $r_{i j}$ and weights $c_{i j}^{q}$ by Eq. (5) and Eq. (6) respectively amounts to changing the speed of molecule by $v_{i j}^{q}(t)$ of Eq. (14).

Proof. Denote the $k$-th terms of Eq. (5) and Eq. (6) by $\left\langle\frac{d r_{i j}(t)}{d t}\right\rangle_{k}$ and $\left\langle\frac{d c_{i j}^{q}(t)}{d t}\right\rangle_{k}$, respectively. When allotted entry $r_{i j}$ is updated according to (5), the first and second terms of (5) will cause the following speed increments of the iteration, respectively: 


$$
\begin{array}{r}
\left\langle d u_{i j}^{q}(t) / d t\right\rangle_{1}^{r}=\frac{\partial u_{i j}^{q}(t)}{\partial r_{i j}(t)}\left\langle\frac{d r_{i j}(t)}{d t}\right\rangle_{1}=\lambda_{1}\left[\frac{\partial u_{i j}^{q}(t)}{\partial r_{i j}(t)}\right]^{2} \\
\left\langle d u_{i j}^{q}(t) / d t\right\rangle_{2}^{r}=\frac{\partial u_{i j}^{q}(t)}{\partial r_{i j}(t)}\left\langle\frac{d r_{i j}(t)}{d t}\right\rangle_{2}=\lambda_{2} \frac{\partial u_{i j}^{q}(t)}{\partial r_{i j}(t)} \frac{\partial J^{q}(t)}{\partial r_{i j}(t)} \\
=\lambda_{2} \frac{\partial u_{i j}^{q}(t)}{\partial r_{i j}(t)} \frac{\partial J^{q}(t)}{\partial u_{i j}^{q}(t)} \frac{\partial u_{i j}^{q}(t)}{\partial r_{i j}(t)}=\lambda_{2} \frac{\partial J^{q}(t)}{\partial u_{i j}^{q}(t)}\left[\frac{\partial u_{i j}^{q}(t)}{\partial r_{i j}(t)}\right]^{2}
\end{array}
$$

Similarly, the third and the fourth term of Eq. (5) will cause the following speed increments of the iteration:

$$
\begin{aligned}
& \left\langle d u_{i j}^{q}(t) / d t\right\rangle_{3}^{r}=-\lambda_{3} \frac{\partial P^{q}(t)}{\partial u_{i j}^{q}(t)}\left[\frac{\partial u_{i j}^{q}(t)}{\partial r_{i j}(t)}\right]^{2} \\
& \left\langle d u_{i j}^{q}(t) / d t\right\rangle_{4}^{r}=-\lambda_{4} \frac{\partial Q^{q}(t)}{\partial u_{i j}^{q}(t)}\left[\frac{\partial u_{i j}^{q}(t)}{\partial r_{i j}(t)}\right]^{2}
\end{aligned}
$$

Similarly, for Eq. (6), we have $\left\langle d u_{i j}^{q}(t) / d t\right\rangle_{k}^{c^{q}}, k=1,2,3,4$. We thus obtain

$$
\begin{aligned}
& \left.\sum_{k=1}^{4}\left[\left\langle d u_{i j}^{q}(t) / d t\right\rangle\right\rangle_{j}^{c^{q}}+\left\langle d u_{i j}^{q}(t) / d t\right\rangle_{j}^{r}\right] \\
& =\left[\lambda_{1}+\lambda_{2} \frac{\partial J^{q}(t)}{\partial u_{i j}^{q}(t)}-\lambda_{3} \frac{\partial P^{q}(t)}{\partial u_{i j}^{q}(t)}-\lambda_{4} \frac{\partial Q^{q}(t)}{\partial u_{i j}^{q}(t)}\right]\left\{\left[\frac{\partial u_{i j}^{q}(t)}{\partial r_{i j}(t)}\right]^{2}+\left[\frac{\partial u_{i j}^{q}(t)}{\partial c_{i j}^{q}(t)}\right]^{2}\right\}=v_{i j}^{q}(t)
\end{aligned}
$$

Therefore, updating $r_{i j}^{(k)}$ and $\left(c_{i j}^{q}\right)^{(k)}$ by (5) and (6), respectively, gives rise to the speed increment of the iteration that is exactly equal to $v_{i j}^{q}(t)$ of Eq. (14).

Theorem 3. Updating the allotted entries $r_{i j}$ and weights $c_{i j}^{q}$ by Eq. (5) and Eq. (6) respectively amounts to increasing the minimal utility of an entry with respect to an objective $q$ in direct proportion to the value of $\lambda_{3}$.

Proof. The speed increment of the iteration, which is related to potential energy $P^{q}(t)$, is given by

$$
\left\langle\frac{d u_{i j}^{q}(t)}{d t}\right\rangle_{3}=\left\langle d u_{i j}^{q}(t) / d t\right\rangle_{3}^{r}+\left\langle d u_{i j}^{q}(t) / d t\right\rangle_{3}^{c^{q}}=-\lambda_{3} \frac{\partial P^{q}(t)}{\partial u_{i j}^{q}(t)}\left\{\left[\frac{\partial u_{j j}^{q}(t)}{\partial r_{i j}(t)}\right]^{2}+\left[\frac{\partial u_{i j}^{q}(t)}{\partial c_{i j}^{q}(t)}\right]^{2}\right\} .
$$

Denote by $\left\langle\frac{d P^{q}(t)}{d t}\right\rangle$ the differentiation of the potential energy function $P^{q}(t)$ with respect to time $t$ arising from using Eqs. (5), (6). We have

$$
\begin{gathered}
\left\langle\frac{d P^{q}(t)}{d t}\right\rangle=\frac{\partial P^{q}(t)}{\partial u_{i j}^{q}(t)}\left\langle\frac{d u_{i j}^{q}(t)}{d t}\right\rangle_{3}=-\lambda_{3}\left[\frac{\partial P^{q}(t)}{\partial u_{i j}^{q}(t)}\right]^{2}\left\{\left[\frac{\partial u_{i j}^{q}(t)}{\partial r_{i j}(t)}\right]^{2}+\left[\frac{\partial u_{i j}^{q}(t)}{\partial c_{i j}^{q}(t)}\right]^{2}\right\} \\
=-\lambda_{3} \omega_{i j}^{2}(t)\left(u_{i j}^{q}(t)\right)^{2} x_{i j}^{2}(t)\left[r_{i j}^{2}(t)+\left(c_{i j}^{q}(t)\right)^{2}\right]\left[u_{i j}^{q}(t)\right]^{2} \leq 0 .
\end{gathered}
$$

where, $\omega_{i j}(t)=\exp \left[-\left(u_{i j}^{q}(t)\right)^{2} / 2 k^{2}\right] / \sum_{i=1}^{I} \sum_{j=1}^{J} \exp \left[-\left(u_{i j}^{q}(t)\right)^{2} / 2 k^{2}\right]$.

It can be seen that using Eqs. (5) and (6) gives rise to monotonic decrease of $P^{q}(t)$. Then by Theorem 1 , the decrease of $P^{q}(t)$ will result in the increase of the minimal utility of entries in distribution vector $R$, in direct proportion to the value of $\lambda_{3}$. 
Theorem 4. The first and second terms of Eqs. (5) and (6) will enable the personal utility of every entry of distribution $R$ to increase, in direct proportion to the value of $\left(\lambda_{1}+\lambda_{2}\right)$.

Proof. According to Eqs. (15) and (16), the sum of the first and second terms of Eq. (5) and (6) will be

$$
\begin{aligned}
& \left\langle d u_{i j}^{q}(t) / d t\right\rangle_{1}^{r}+\left\langle d u_{i j}^{q}(t) / d t\right\rangle_{2}^{r}+\left\langle d u_{i j}^{q}(t) / d t\right\rangle_{1}^{c^{q}}+\left\langle d u_{i j}^{q}(t) / d t\right\rangle_{2}^{c^{q}} \\
& =\left[\lambda_{1}+\lambda_{2} \frac{\partial J^{q}(t)}{\partial u_{i j}^{q}(t)}\right]\left\{\left[\frac{\partial u_{i j}^{q}(t)}{\partial r_{i j}(t)}\right]^{2}+\left[\frac{\partial u_{i j}^{q}(t)}{\partial c_{i j}^{q}(t)}\right]^{2}\right\}=\left(\lambda_{1}+\lambda_{2}\right) x_{i j}^{2}(t)\left[r_{i j}^{2}(t)+\left(c_{i j}^{q}(t)\right)^{2}\right]\left[-u_{i j}^{q}(t)\right]^{2} \\
& \geq 0 .
\end{aligned}
$$

Therefore, the first and second terms of (5) and (6) will cause $u_{i j}^{q}(t)$ to monotonically increase.

Theorem 5. Updating $r_{i j}$ and $c_{i j}^{q}$ by Eqs. (5) and (6) gives rise to monotonic increase of the whole utility of all the entries of distribution $R$, in direct proportion to the value of $\lambda_{2}$.

Proof. Similar to Theorem 4, it follows that when an entry $(i, j)$ modifies its $r_{i j}$ and $c_{i j}^{q}$ by Eqs. (5) and (6), differentiation of $J^{q}(t)$ with respect to time $t$ will not be negative -i.e., $\left\langle\frac{d J^{q}(t)}{d t}\right\rangle \geq 0$, and it is directly proportional to the value of $\lambda_{2}$.

Definition 9. (Max-min Fairness) A feasible distribution $R$ is max-min fair if and only if an increase of any entries of distribution vector $r$ within the domain of feasible distributions must be at the cost of an decrease of some already smaller entries $r$. Formally, for any other feasible distribution $Y$, if $y_{i j}>r_{i j}$ then there must exist some $\left(i^{\prime}, j^{\prime}\right)$ such that $r_{i^{\prime} j^{\prime}} \leq r_{i j}$ and $y_{i^{\prime} j^{\prime}}<r_{i^{\prime} j^{\prime}}$.

Theorem 6. (Max-min fair allocation) Max-min fair solution with multi- objective can be obtained by updating the allotted entries $r_{i j}$ and weights $c_{i j}^{q}$ by Eq. (5) and Eq. (6) respectively.

Proof. It is straightforward from Theorems 1-5 and Definition 9.

Theorem 7. Updating $r_{i j}$ and $c_{i j}^{q}$ by Eqs. (5) and (6) gives rise to monotonic decrease of the interaction energy $Q^{q}(t)$, in direct proportion to the value of $\lambda_{4}$.

Proof. As in the above, we have

$$
\begin{aligned}
& \left\langle\frac{d u_{i j}^{q}(t)}{d t}\right\rangle_{4}=-\lambda_{4} \frac{\partial Q^{q}(t)}{\partial u_{i j}^{q}(t)}\left\{\left[\frac{\partial u_{i j}^{q}(t)}{\partial r_{i j}(t)}\right]^{2}+\left[\frac{\partial u_{i j}^{q}(t)}{\partial c_{i j}^{q}(t)}\right]^{2}\right\} ; \text { and } \\
& \left\langle\frac{d Q^{q}(t)}{d t}\right\rangle=\frac{\partial Q^{q}(t)}{\partial u_{i j}^{q}(t)}\left\langle\frac{d u_{i j}^{q}(t)}{d t}\right\rangle_{4}=-\lambda_{4}\left[\frac{\partial Q^{q}(t)}{\partial u_{i j}^{q}(t)}\right]^{2}\left\{\left[\frac{\partial u_{i j}^{q}(t)}{\partial r_{i j}(t)}\right]^{2}+\left[\frac{\partial u_{i j}^{q}(t)}{\partial c_{i j}^{q}(t)}\right]^{2}\right\} \leq 0 .
\end{aligned}
$$

Updating $r_{i j}$ and $c_{i j}^{q}$ by Eqs. (5) and (6) makes the interaction energy $Q^{q}(t)$ smaller and smaller. Thus it is possible to satisfy the restrictions.

Theorem 8. The MMA can solve the distribution problem defined in Definition 1.

Proof. It is straightforward from Theorems 6-7. 


\section{Simulation}

Because the problem-related matrices are too large to be listed in this paper, we go directly to the results of these two problems. The experimental evolutionary results for $z$ from $t=1$ to $t=1000$ are depicted in Fig. 1 .

As shown in Fig. 1, Curve A, at $t=983, z$ reaches its maximum, and stays unchanged in the remainder of the iterations; for Curve $\mathrm{B}$, at $t=968, z$ reaches its maximum, and stays unchanged in the remainder of the iterations. At the two points, the MMA converges to a stable equilibrium state and produces the optimum solutions. The results confirm the usefulness of MMA for large-scale NP-hard combinatorial optimization problems.

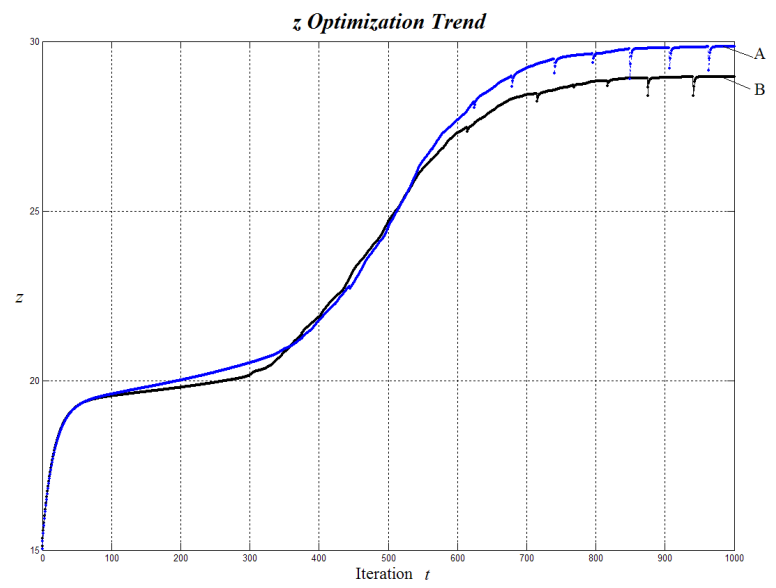

Curve A: Social behaviors not involved

Curve B: Social behaviors involved

Fig. 1. Comparison of $z$ optimization in large-scale problems with social behaviors involved and not involved

\section{Motivation and Biological Meaning of MMA}

In the MMA, all the entries in distribution vector $R$ are treated as molecules which are located in their own force fields. This transforms the distribution problem into the kinematics and dynamics of the molecules in a set of force fields. In molecular mechanics, Newtonian mechanics is used to model molecular systems and force fields are used to calculate the potential energy.

MMA puts emphasis on

- providing a view of individual and whole optimization (with one to two objectives);

- parallelization with reasonably low time complexity;

- all multiple objectives being optimized individually as well as collectively;

- the ability to deal with social interactions;

- the biological meaning of the model. 
In the biological model of MMA, there are $I \times J$ molecules (every entry in distribution vector $R$ is treated as a molecule), and the same number $(I \times J)$ of force fields. These force fields are evenly distributed in the horizontal plane. Every molecule moves in its own force field. When the number of optimization objectives is $Q$, the force fields of all molecules form $Q$ dimensions where the coordinates in the space are in $[0,1]$. In the biological model of MMA, $s_{i j}$ represents molecule $(i, j)$.

If the number of maximum objectives is 1 , the molecules will move upwards on one-dimensional spaces (lines) $\left(u_{i j}^{1} \in[0,1]\right)$ during the optimization process. If the number of maximum objectives is 2 , the molecules will move away from the origin in two-dimensional spaces (planes) $\left(u_{i j}^{1} \in[0,1], u_{i j}^{2} \in[0,1]\right)$ during the optimization process. Analogously, if the number of maximum objectives is $Q$, the molecules will move away from the origin in $Q$-dimensional spaces $\left(u_{i j}^{1} \in[0,1], \cdots, u_{i j}^{q} \in[0,1], \cdots, u_{i j}^{Q} \in[0,1]\right)$ during the optimization process, where $u_{i j}^{q}$ is a coordinate of the $q$-dimensional space.

Molecules in the MMA move not only under outside forces, but also under their internal force; hence they are different from molecules in molecular mechanics. In fact, the evolution of $s_{i j}$ involves two variables $-r_{i j}, c_{i j}^{q} . r_{i j}$ and anyone of $c_{i j}^{q}(q=1, \cdots, Q)$ are reciprocal dual.

In a force field $F_{i j}$, the coordinates of the $Q$-dimensional space of the molecules represent the utilities with respect to the $q$-th objective of the entry $(i, j)$ of the distribution vector $R$ that is described as a molecule. A molecule will be influenced simultaneously by several kinds of forces in the $Q$-dimensional space, which include the gravitational force of the $Q$-dimensional space force field where the molecule is located, the pulling or pushing forces stemming from the interactions with other molecules, and the molecule's own autonomous driving force.

When the number of maximum objectives is 1 , that is, a single objective optimization problem, all the above-mentioned forces that are exerted on a molecule are dealt with as forces along a vertical direction (along a line). Thus a molecule will be driven by the resultant force of all the forces that act on it upwardly or downwardly, and move along a vertical direction. The larger the upward resultant force on a molecule, the faster the upward motion of the molecule. When the upward resultant force on a molecule is equal to zero, the molecule will stop moving, being at an equilibrium status. As shown in Fig. 2, the molecules move in their own one-dimensional force fields $F_{i j}$ (lines).

The upward gravitational force of a force field on a molecule causes an upward component of the motion of the molecule, which represents the tendency that the molecule pursues the common benefit of the whole. The upward or downward component of the motion of a molecule, which is related to the interactions with other molecules, depends upon the strengths and categories of the interactions. The molecule's own autonomous driving force is proportional to the degree the molecule tries to move upwards in its own force field where it is located, i.e., the molecule tries to acquire its own maximum utility.

When the number of maximum objectives is 2, each molecule moves away from the origin in its own force field (a unit plane), as shown in Fig. 2. 


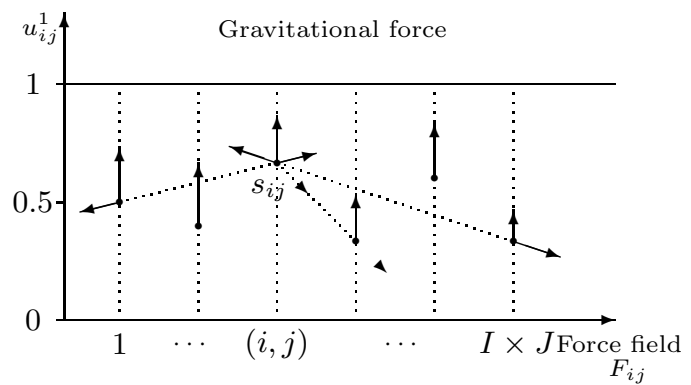

Fig. 2. The biological model of MMA for single objective optimization

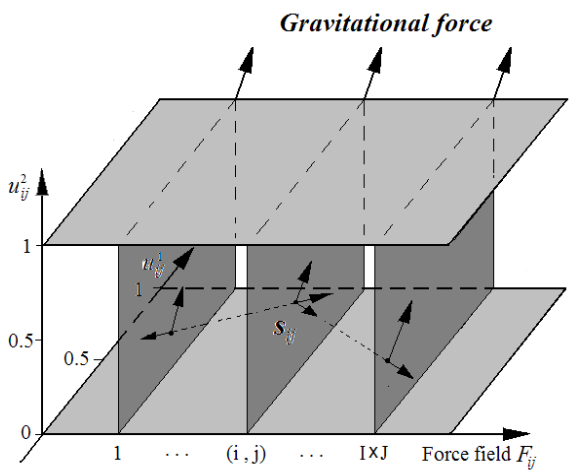

(a) A 3D global view of MMA

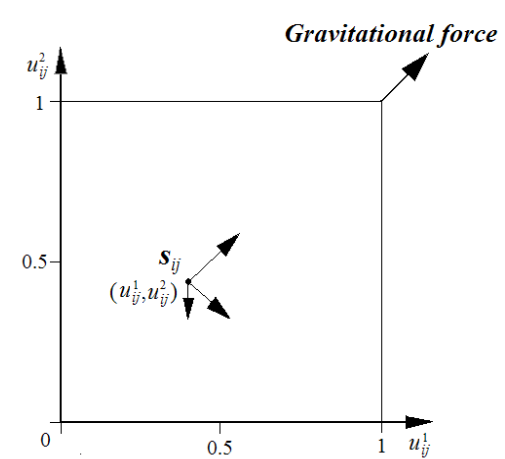

(b) Force field $F_{i j}$

Fig. 3. The biological model of MMA for two objectives optimization

When the number of maximum objectives is $Q>2$, each molecule moves away from the origin in its own $Q$-dimensional force field. $Q$-dimensional force field and $Q+1$-dimensional biological model of MMA are abstract mathematical spaces.

One major difference between the molecule of the proposed MMA and the molecule of a classical biological model is that the molecule in MMA has its own driving force which depends upon the autonomy of the molecule. All the molecules, in their own $Q$-dimensional force fields, simultaneously, evolve under their exerted forces; as long as they gradually reach their equilibrium positions from their initial positions which are set at random, we can obtain a feasible solution to the multiple objectives distribution problem.

There are three main components of the MMA biological model. We call them as "skeleton", "muscle" and "blood".

\footnotetext{
Molecules motion-skeleton

How to move (mathematical model of MMA)—muscle

Energy (energy function) -blood
} 


\section{In summary}

- Every entry in distribution vector $R$ is treated as a molecule.

- In Section II, we defined some concepts in kinematics and dynamics based on both biology ideas and our optimization problem.

- We defined energy function as

Energy $=$ personal utility kinetic energy

+ whole utility kinetic energy

+ max-min fair gravitational potential energy

+ interaction potential energy

It is difficult to say which one occurred to us earlier: the idea of the mathematical model of MMA or the idea of the biological model. We found inspiration in and a foundation from molecular mechanics, leading to the proposal of the mathematical model of MMA. Both the mathematical model and the biological model have a system of their own. There are the correspondences between them.

\section{Conclusion}

In this paper, we propose a novel Molecular Mechanics Algorithm (MMA) as a new branch of nature-inspired algorithms for solving multi-objective NP-hard combinatorial optimization problems. The MMA is inspired by the biological model of molecule dynamics. The approach maps a given combinatorial optimization problem to the motion of molecules in the corresponding multi-dimensional force fields. The molecules move according to certain rules defined by a mathematical model until arriving at a stable state; subsequently, the solution of the multi-objective combinatorial optimization problem is obtained by anti-mapping the stable state. We have discussed the mathematical model, algorithm, motivation, biological model, and the experiments of the MMA in detail.

The MMA can work out the theoretical optimum solution, which is important and exciting. We have given the theoretical proofs and experiments to verify this key point.

Although there are many differences between molecules in molecular mechanics and those in the MMA, we have shown that being inspired by molecular mechanics, the MMA enables feasible multi-objective optimization in very large scales. The MM approach can work out the theoretical optimum solution and has a low computational complexity, which is crucial for the functioning of largescale NP-hard combinatorial optimization problems.

Acknowledgements. This project is supported by the Hong Kong General Research Fund under Grant No. HKU 713708E, the National Science Foundation of China (NSFC) under Grant No. 60575027, the High-Tech Development Program of China (863) under Grant No. 2006AA10Z315, and the Specialized Research Fund for the Doctoral Program of Higher Education under Grant No. 20060251013. 


\section{References}

1. Shadbolt, N.: Nature-Inspired Computing. IEEE Intelligent Systems 19, 1-2 (2004)

2. Holland, J.: Adaptation in Natural and Artificial Systems. MIT Press, Cambridge (1976)

3. Dorigo, M.: Optimization, Learning and Natural Algorithms. Ph.D. Thesis, Dipartimento di Elettronica, Politecnico di Milano, Italy (1992) (in Italian)

4. Dorigo, M., Maniezzo, V., Colorni, A.: Ant System: Optimization by a Colony of Cooperating Agents. IEEE Trans. Systems, Man, Cybernet., Part B 26(1), 29-41 (1996)

5. Kennedy, J., Eberhart, R.: Particle Swarm Optimization. In: IEEE Conf. Neural Networks, pp. 1942-1948. IEEE Press, Los Alamitos (1995)

6. Kirkpatrick, S., Gelatt, C., Vecchi, M.: Optimization by Simulated Annealing. Science 220(4598), 671-680 (1983) 